\title{
Discursos de las memorias colectivas. Reflexiones metodológicas acerca de la reconstrucción del recuerdo en grupos de discusión
}

\author{
Bruno Miguel Carriço Reis
}

\author{
Departamento de Ciências da Comunicação, Universidade Autónoma de Lisboa, Portugal I \\ breis@autonoma.pt I https://orcid.org/0000-0001-6420-8781
}

\begin{abstract}
Resumen: El presente texto pretende contribuir a la reflexión sobre los usos del grupo de discusión como instrumento metodológico que permite la reconstrucción de las memorias colectivas. Se discuten los principales desafíos en la lectura y sistematización de los discursos producidos desde nuestra experiencia con los grupos de discusión. Desde 2004 hemos llevado a cabo más de 50 grupos de discusión en Portugal y España para intentar comprender qué recuerdos se evocan y cómo se elaboran las memorias de las diferentes capas de la población en relación con las dictaduras y las transiciones de estos dos países ibéricos. En concreto, comparamos las "memorias vividas" (actores socializados en la experiencia dictatorial y que fueron testigos de la transición a la democracia) con las "memorias mediadas" (actores que reconstruyen el pasado utilizando testimonios o representaciones sociales de la dictadura/transición). Este artículo muestra que la reconstrucción del pasado, a través de los diálogos construidos en los grupos de discusión, es una tarea epistemológicamente desafiante, ya que vincula la necesidad del investigador de articular los relatos producidos con la realidad histórica que les dio sentido en un proceso hermenéutico de la conciencia
\end{abstract} histórica.

Palabras clave: Grupos de Discusión; Memoria Colectiva; Metodología Cualitativa; Discursos Sociales.

\section{Discourses of Collective Memory. Methodological Reflections on the Reconstruction of Remembrance Using Focus Groups}

\begin{abstract}
The present text aims to contribute to the reflection about the uses of focus groups as methodological instruments that permit the reconstruction of collective memories. The main challenges in reading and systematizing the discourses produced from the research practice constitute the main topics of discussion in this research. Since 2004 over 50 discussion groups have been facilitated both in Portugal and Spain in order to try to understand which memories were invoked and how the memories of different layers of the population were elaborated in relation to the dictatorships and transitions. Specifically, the "lived memories" (actors socialized in the dictatorial experience who witnessed the transition to democracy) and the "mediated memories" (actors who reconstructed the past using testimonials or social representations of the dictatorship/transition) were object of comparison. This article shows that the reconstruction of the past through the dialogues produced in the discussion groups is an epistemologically challenging task whereby the researcher is called upon to articulate the accounts produced with the historical reality where meaning was first produced, in a hermeneutic process of historical consciousness.
\end{abstract}

Keywords: Focus Groups; Collective Memory; Qualitative Methodology; Social Discourses.

\section{Introducción}

En este trabajo mencionamos los retos de la reconstrucción de la memoria como proceso social a partir de los discursos recogidos en grupos de discusión, partiendo de nuestra amplia experiencia con este procedimiento metodológico. Hemos realizado 50 grupos de discusión (entre 2004 y 2019), con los que quisimos demostrar que las memorias se asocian a las dictaduras y transiciones portuguesa y española. El objetivo central que pretendemos con esta reflexión es discutir sobre los retos metodológicos que supone interpretar los discursos producidos en los grupos de discusión. Concebimos el lenguaje como una herramienta esencial para dar sentido al mundo social; al igual que Ricoeur (1999, 2003), que definió el orden lingüístico como dependiente y no autónomo, lo que remite a una experiencia del mundo. El lenguaje es un actor social que se articula mediante el discurso, y la memoria es una práctica social discursiva que depende del carácter lingüístico para su transmisión (Ormeño, 2004). 
Esta premisa afirma la importancia substancial del lenguaje respecto a la memoria colectiva, un elemento que Halbwachs consideró como "el marco más elemental y a la vez más estable de la memoria" (2004, p.10). Partimos de esta idea con la intención de construir instrumentos metodológicos que nos ayuden en la definición de las estrategias interpretativas para convertir los actos de habla de los participantes en interpretaciones que configuren tipificaciones de las memorias colectivas estudiadas, como demostraremos en la parte final de este texto.

No olvidamos que la interpretación de los resultados obtenidos a partir de las conversaciones en los grupos de discusión depende de la forma en que se organicen y dinamicen las conversaciones, especialmente en lo que se refiere a los siguientes aspectos: al punto de partida epistemológico y metodológico adoptado por el investigador ${ }^{1}$ (Crotty, 1998); a la manera en que se forma y convoca el grupo²; a las condiciones materiales en que se desarrolla la sesión ${ }^{3}$; a la actitud del moderador en la gestión de la conversación, a la propia dinámica del grupo y del guion propuesto ${ }^{4}$; al número de participantes ${ }^{5}$; al estímulo utilizado ${ }^{6}$; al número de grupos realizados ${ }^{7}$; a la transcripción y modelo de análisis definido para guiar la interpretación de los resultados ${ }^{8}$. Para profundizar en estos aspectos recomendamos los trabajos de Hennink (2014) y de Gutiérrez (2008). Pero nuestra propuesta va en otra dirección.

Desde los años noventa, autores como Bourdieu y Wacquant (1992) y Ritzer (1992) vienen advirtiendo de la necesidad de que la sociología se vuelva reflexiva. Esta afirmación puso de manifiesto la importancia de que los sociólogos se replanteen sus prácticas de teorización y el modo en que se llevan a cabo los proyectos metodológicos.

\footnotetext{
${ }^{1}$ En nuestro caso concreto, partimos de una orientación de constructivismo social, deduciendo el sentido de la percepción del pasado dictatorial a través de los significados atribuidos a este tiempo histórico por los participantes de los grupos de discusión, como manifestación de la(s) memoria(s) colectiva(s) producida(s) sobre este tiempo histórico. Leemos los discursos que surgen a través de la comprensión hermenéutica propuesta por Gadamer (1989), mediante el sentido atribuido a los significados históricos.

${ }^{2}$ Nuestra estrategia de convocatoria consistió en movilizar a miembros de grupos ya constituidos socialmente (grupos de amigos, miembros de sindicatos, partidos políticos, asociaciones de vecinos, colectivos feministas, etc.). Hemos formulado una orientación que llamamos "grupos naturales". Para la comprensión de este posicionamiento metodológico se recomienda la lectura de la fundamentación realizada en el texto de Carriço Reis (2009). Conformamos grupos homogéneos y heterogéneos en cuanto a: género, edad, clase social, contexto geográfico, grado de politización para comprobar la preponderancia de cada una de estas variables en la forma de construir las percepciones del pasado.

${ }^{3}$ De acuerdo con la nota anterior, realizamos los grupos de discusión en los contextos sociales recurrentes, intentando así "naturalizar" la conversación.

${ }^{4}$ La actitud adoptada fue de total adaptación a la dinámica desarrollada por los grupos. Optamos por una posición no directiva, interviniendo sólo en aquellos momentos en los que registrábamos una ruptura en la dinámica de la conversación, pero siempre para dar seguimiento a las propuestas temáticas planteadas por los interlocutores (ya que era importante para nosotros saber qué significaba la iniciativa de los participantes desde el punto de vista memorialista). Nuestro guion fue abierto, explorando todas las posibilidades presentadas por los interlocutores.

${ }^{5}$ Optamos preferentemente por respetar el número de participantes de los grupos ya constituidos socialmente, siempre que no fuera extenso (no más de 9 miembros), para asegurar una dinámica que permitiera la participación de todos ellos en el diálogo.

${ }^{6}$ En los grupos celebrados en Portugal y España utilizamos el mismo estímulo, el episodio de la primera temporada de la serie Cuéntame..., titulado Una larga espera / Uma larga espera, ya que en ambos países la serie supuso un éxito de audiencias. La versión original española fue adaptada al contexto portugués de la dictadura y transición, y dadas las similitudes de los procesos históricos la mayoría de los episodios son de enorme similitud. El episodio elegido es de hecho un ejemplo, idóneo para posteriores lecturas comparativas entre las interpretaciones generadas entre los grupos de portugueses y españoles. Dicho episodio supuso el punto de partida de los grupos de discusión en cada uno de los países estudiados. La utilización de este capítulo como paso previo al inicio del debate se debe: 1) a la necesidad de contextualizar las épocas que queremos que sean el epicentro de la discusión, la dictadura y transición. Gracias a ello clasificamos y acotamos, de forma no manifiestamente dirigida, los temas de debate. De igual modo, dicho capitulo sirve 2) como punto de arranque para activar otras memorias mediáticas que puedan estar asociadas.

${ }^{7}$ Utilizamos el criterio del punto de saturación para determinar el número de grupos considerados en cada estudio de investigación. Dicho procedimiento obliga a los investigadores a transcribir y analizar de inmediato los discursos producidos al final de cada grupo, con el fin de comprender los temas abordados y en qué medida cada grupo plantea aspectos reveladores en relación con los grupos establecidos anteriormente. En el momento en el que no se dice nada relevante, se da fin a estos grupos de discusión.

${ }^{8}$ En nuestro caso hemos tenido en cuenta los marcos sociales de la memoria de Maurice Halbwachs (2004).
} 
Sin embargo, el llamamiento a producir una "sociología de la sociología", por utilizar el concepto acuñado por Bourdieu, es un ejercicio de segundo orden en el debate académico. La "obsesión empírica", como sello de la industrialización de la publicación académica, ha restado espacio a una amplia tradición intelectual sociológica que se apoyó desde sus inicios en la discusión de sus procedimientos ${ }^{9}$.

Del mismo modo, los manuales de investigación social más populares ${ }^{10}$ se centran especialmente en explicar los métodos y las técnicas como una prescripción de procedimientos mecánicos y no destacan en la discusión epistemológica el uso de las herramientas metodológicas y la labor de la interpretación de los datos.

Observamos un importante esfuerzo de los sociólogos por problematizar sobre los usos de los grupos focales, pero esencialmente los centrados en los diseños de investigación, su conducción y las dinámicas generadas en las conversaciones colectivas, pero hay pocos estudios que se dediquen a decodificar la reconstrucción de los discursos producidos, como señalan enfáticamente los trabajos de Pearson y Vossler (2016), Gutiérrez de Álamo (2009) y Wilkinson (2004).

Nuestro esfuerzo seguirá por eso la línea de las aportaciones que intentan reflexionar acerca de cómo los científicos sociales operan delante de los discursos producidos, en nuestro caso concreto de enunciados portadores de "conciencias históricamente realizadas"11 (Gadamer, 1989, p. 341). La hermenéutica histórica propuesta por el autor plantea que el significado del tiempo es una construcción derivada de una lógica de mediación y que el del presente es ya un significado del pasado, importando entender cómo se consolidó, ya que "recuperamos los conceptos de un pasado histórico de tal manera que también incluyen nuestra propia comprensión de ellos" (Gadamer, 1989, p. 374). Es dar sentido al significado dado por quienes lo interpretan, pero diluyendo el efecto subjetivo de estas lecturas, ya que el significado de la historia es un atributo construido socialmente (familia, sociedad, corporaciones) que debe ser reconstruido desde estas premisas socializadoras que el investigador debe decodificar. Gadamer recupera el sentido que Maurice Halbwachs (2004) atribuye a los marcos de la memoria como los "elementos determinantes que posibilitan toda construcción de la memoria en su sentido social, mediante un sistema de valores (de ahí la importancia de los procesos de socialización) que unifican los pensamientos individuales y los insertan en la memoria colectiva" (Carriço Reis, 2009, p. 42).

Por eso, "una actividad en apariencia tan libre, desvinculada de restricciones, reglas y procedimientos, como es el acto de conversar, el 'decir cuatro cosas', se presenta, en realidad, como el lugar donde se ejecutan competencias socialmente adquiridas y relevantes" (Wolf, 2000, p. 186). El lenguaje no sólo es un sistema normativo gramatical, sino una construcción social manifestada de diferentes maneras, un "juego lingüístico" según la definición de Wittgenstein (2009, p.23). En definitiva, las palabras son una condición social básica con un elevado trasfondo social (Bourdieu, 2001), detenernos en los enunciados de los recuerdos/olvidos acerca de los autoritarismos ibéricos es percibir que el pasado se hace presente, partiendo del interrogante: ¿los discursos producidos qué memorias colectivas tipifican?

\footnotetext{
${ }^{9}$ El texto de Cyntia Hamlin; Breve Metametodología das Ciências Sociais (2011), hace una interesante síntesis del camino recorrido por la reflexión sociológica y señala la inflexión de este ejercicio meta-metodológico.

${ }^{10}$ Basta con hojear las propuestas de autores como Raymond Quivy \& Luc Van Campenhoudt, Judith Bell y Umberto Eco, por citar los bestsellers metodológicos más destacados.

${ }_{11}$ Nuestra traducción.
} 


\section{Los marcos Discursivos de la Memoria. Reflexiones Teórico-Metodológicas de Cómo Darle Sentido Interpretativo al Pasado}

Los grupos de discusión sociológicamente contextualizados son los espacios de significación donde intentamos reproducir un discurso social representativo de un determinado enunciado.

Para ello, tendremos en cuenta que la memoria, incluso desde el pensamiento colectivo, es un elemento invisible ubicado en las dinámicas internas de los grupos. Por este motivo, la constitución de los grupos de discusión no se reduce simplemente a lo micro social, sino que atiende a "una situación micro social -la reunión-, con una aplicación macrosocial" (Callejo, 2001, p. 22). Esta técnica nos permite "analizar la acción-en-contexto, dado que no sólo existe el concepto de contexto-en-general, sino que todo uso de 'contexto', sin excepción, es en sí mismo contextual" (Garfinkel, 2006, p. 19). Esta es la fórmula empleada para "comprender el pasado por el presente" (Bloch, 2001, p. 38), ya que cada individuo "sólo es accesible desde su interioridad dentro de su interrelación con otros individuos" (Dilthey, 2000, p. 184).

El habla crea realidad (Murillo y Mena, 2006). La construcción autobiográfica que hace el sujeto cuando toma la palabra dentro del grupo de discusión se acercará a su condición colectiva en la medida en que se significa siempre con otros. Este hecho, en sí mismo, ya expresa una identidad, como señala Dilthey (2000), al describir una historia con base en la coherencia y la relación entre los hechos.

Partiendo de que el discurso nunca es individual -no existe subjetivación plena-, nuestra finalidad se orienta a buscar una realidad socio-histórica conectada con el individuo que habla. La organización de la memoria se vincula, en un primer momento, a nuestra identidad personal, pero "el 'yo' es el resultado del acto de hablar sobre nosotros mismos" (Duero, 2006, p. 138). Así, la biografía de los participantes del grupo indica dónde se produce el discurso social (Callejo, 2001). Sin embargo, los datos los obtenemos a partir de la interacción; por lo que seguimos una lógica dialógica y no de producción de discursos individuales. Es así como el lenguaje produce una dinámica social al ubicarse como intersubjetividad, generando unidad al producir una afinidad en la forma de pensar, o sea, en los enunciados del habla. El sujeto se siente así reconocido en el mensaje (Laughey, 2006). Por eso, los significados sociales emergen de la interacción verbal, como postula Garfinkel (2006).

El lenguaje es la expresión de nuestros pensamientos desde una posición social. De este modo, contextualizar los discursos da "valor de supervivencia al acontecimiento" (Ibáñez, 1985, p. 131). Este hecho llevó a Pierre Bourdieu a preguntarse: ¿Qué significa hablar? (2001), ya que el lenguaje nos remite a la estructura al representar esta en su propia forma (Garfinkel, 2006). Por tanto, reconocer un modo de hablar posibilita construir el discurso de un individuo, del que sabemos su estructura social. La teoría de la distinción que desarrolló Bourdieu (2006) nos ayuda a averiguar desde qué posición hablan los individuos en los grupos de discusión.

Partiendo de los conceptos de campo y habitus propuestos por Pierre Bourdieu (2006), podemos construir las ubicaciones objetivas y subjetivas que construyen el lenguaje "porque no sólo 'los significados hacen a los sujetos' (...) sino que también los sujetos hacen a los significados" (Pérez-Agote, 2008, p. 40). Los individuos, en su proceso de rutina cotidiana, reproducen su habitus. Este habitus está directamente relacionado con el lenguaje porque genera un contexto de interacción social, al modo de un juego de fuerzas correspondientes a la situación social de cada colectivo; es decir, de su posición de campo. Por tanto, el discurso es una articulación de una realidad objetivamente subjetivada, donde el poder establecido fuera de la interacción -según su capital simbólico- determina lo que va a ocurrir en la interacción. 
"No podemos imaginarnos una trayectoria sin construir previamente los estados sucesivos de su campo de desarrollo ni, por tanto, del conjunto de relaciones objetivas del agente asociado -o por lo menos, en cierto número de estados pertinentes- al conjunto de agentes involucrados en el mismo campo y confrontados con las mismas posibilidades" (Bourdieu, 2006, p. 190).

Para saber si existe una memoria colectiva similar en diferentes grupos, pero de la misma estructura social, se debe producir en marcos sociales de memoria equiparable. Por tanto, debemos conocer previamente cómo interactúan desde su posición en la estructura social. Para ello, analizaremos cómo los enunciados subjetivos se relacionan con una lógica social que depende de las prácticas objetivas -el campo- y que modela al agente que se incorpora a un discurso.

Este enunciado permite a los investigadores reconstruir una realidad y una visión del mundo, es decir, un habitus o una "subjetividad socializada". "El discurso, en cuanto reconstrucción de las experiencias por parte de los sujetos, otorga objetividad a lo que en un primer momento puede parecer subjetivo y, por lo tanto, intransmisible" (Callejo, 1995, p. 9).

Cada vez es más difícil establecer una relación directa entre las posiciones objetivas que los grupos ocupan en la estructura social, porque el entrecruzamiento de varios lenguajes acrecentó la complejidad de la propia acción discursiva de los grupos (Vala, 2000). Antes, a cada memoria colectiva le correspondía una forma particular de representar el pasado, basándose en una retórica característica (Bourdieu, 2001); ahora, se señala un cambio significativo provocado por el advenimiento de la sociedad digital. Este cambio se materializó en una socialización pautada por una dependencia mediática (Hoskins, 2018). En ella, los discursos se tejen mediante distintos lenguajes que se entrecruzan, lo que dificulta la tarea de encontrar los "discursos tipo"; esos repertorios que son el conjunto de elementos comunes a una determinada manera de pensar (Laughey, 2006). De este modo, nos mostramos ante los demás mediante el acto del lenguaje (Bruner, 2003). No obstante,

el lenguaje codifica. Esta codificación puede ser realmente perceptible, emergiendo de nuestras memorias compartidas, aspiraciones históricas, circunstancias políticas y sociales. Sin embargo, también puede simular necesidades y significaciones esenciales, individualizadas e intensamente privadas. El lenguaje es, en sí mismo y por sí mismo, plurilingüe. Contiene mundos (Steiner, 2008, p. 95).

En medio de este entramado complejo de múltiples significados, sólo una perspectiva metodológica con una dinámica intertextual, que relacione los distintos discursos de la memoria colectiva, puede llegar al llamado manto adjetival. Este último es un acto de habla que responde a un yo social determinado (Heller, 2003). Es decir, esas "memorias comunicativas" de las que habla Assmann (1995, p.126), son construcciones del lenguaje sobre hechos pasados que proponen apropiaciones capaces de crear sentido colectivo, ya que "las representaciones sociales circulan en discursos" (Callejo, 2001, p. 48).

Hacer lecturas del pensamiento colectivo es cada vez una tarea más compleja, ya que los discursos se dejaron permear por una sobreabundancia informativa, crearon "nuevas memorias cosmopolitas" y globales (Levy y Sznaider, 2002, p. 88). Lo que llevó, a algunos autores, a considerar que estamos más allá del giro lingüístico, en estos momentos en los que la amplitud de la comunicación digital ahora no deja sobrevivir al relato por sí mismo, como testimonio. Esto se debe a su formato híbrido. Tenemos que orientar nuestro esfuerzo hacia los elementos que componen el discurso, por lo que llegamos al giro interpretativo. "La reflexión contemporánea en torno a lo social ha superado los avances que en su momento supuso el giro lingüístico de las ciencias sociales. Hoy, por las características específicas de las sociedades globales -ambivalencia, complejidad, riesgolos investigadores sociales se esfuerzan en entender y explicar los mecanismos de conformación de identidades cada vez más complejas" (Arias, 2002, p. iv). 
La problemática del lenguaje se centra en sus límites. Determinar la efectividad de su uso presenta el doble problema de decir lo que realmente se quería decir para que "la palabra que alcanza el otro" sea la misma que comunique nuestra intención; tal y como corroboró Gadamer (1989).

El giro interpretativo reivindica sin tapujos la posibilidad de superar la complejidad dada por la supremacía del intérprete sobre el actor, tal y como descubrió Schleiermacher (1990), porque "la hermenéutica dejó de ser un análisis filológico de los textos remanentes de otros escritores: se convirtió en el problema de un miembro de una cultura esforzándose por captar la experiencia de otro" (Bauman, 2002, p. 27). Para Steiner a este esfuerzo le corresponde siempre, por norma, un juicio de valor; "el acto y el arte de la lectura seria conllevan dos movimientos principales; del espíritu: interpretación (hermenéutica) y valoración (crítica, juicio estético). Ambos movimientos son estrictamente inseparables. Interpretar es juzgar" (1997, p. 51).

Por los motivos que alega Gadamer en su obra verdad y método (1989), la interpretación requiere un "cegamiento parcial". La cuestión no es nueva; recupera el debate epistemológico previo de la neutralidad de investigador al investigado, cuando se acerca a lo que ese quiere decir. La diferencia primordial que postuló Dilthey (2000) en su momento, lo llevó a establecer la distinción ya clásica entre "explicar" (Erklären) y "comprender" (Verstehen). Esto es, "mientras que las ciencias puras buscan explicar los fenómenos a partir de hipótesis y leyes generales, las ciencias del espíritu quieren comprender una individualidad histórica a partir de sus manifestaciones exteriores. La metodología de las ciencias del espíritu será de esta manera una metodología de la comprensión" (Grondin, 2008, p. 39). Estos planteamientos merecieron la crítica y el desacuerdo de Max Weber. El sociólogo alemán reflexionó sobre este planteamiento proveniente de la Escuela Histórica en sus escritos: Sobre algunas categorías de la sociología comprensiva, datados originalmente en 1913. Max Weber propone un planteamiento que acabe con esa tensión y que dote a la sociología de los elementos capaces de construir leyes verificables empíricamente sobre el "actuar racional de acuerdo con fines". Este planteamiento permitiría dotar de sentido la acción.

El problema es que "la comprensión, como la etimología indica, 'comprehende' no solamente en términos de conocimiento, sino también de cerco y de injerencia" (Steiner, 1997, p. 337). Por eso, el acto de entendimiento tiene que revelarnos, mediante una práctica metodológica sustentada en la empírica, el porqué de una determina acción. Para llevar a cabo esta labor analítica en los grupos de discusión optaremos por determinar cómo esas acciones lingüísticas hacen de mediación de las interacciones sociales (Winch, 1972). Ya que "a este proceso, por el cual conocemos el interior de los individuos a partir de signos dados sensiblemente desde fuera, lo llamamos: comprender" (Dilthey, 2000, p. 25). En el apartado final de texto discurriremos acerca de las opciones interpretativas para darle sentido comprehensivo a los discursos que recogemos en los grupos de discusión.

\section{A Modo de Conclusión: Praxis Analítica para una Tipificación de las Memorias Colectivas de las Dictaduras y Transiciones Ibéricas}

Resumiendo, nuestro esfuerzo se orientó a recoger un corpus discursivo capaz de determinar la particularidad de dicho enunciado, como acciones racionales que construían tipologías del recuerdo, donde "los tipos no son yuxtapuestos, sino derivados sistemáticamente con respuestas alternativas a un determinado problema" (Poggi, 2005, p. 35). Buscamos recoger los discursos que nos parecieron relevantes, ya que en las "técnicas cualitativas basamos la representatividad en principios estructurales: no nos interesa saber cuántos mantienen un determinado discurso, sino cómo se articulan internamente los diferentes discursos existentes sobre la realidad, que es nuestro objeto de estudio" (Murillo y Mena, 2006, p. 100). 
No buscamos una representatividad proporcional del universo de los actores sociales, más si por medio de los grupos de discusión construir enunciados representativos del pensar de la(s) memoria(s) colectiva(s) para el caso portugués y español. Nuestra finalidad es buscar los discursos tipo, en el sentido weberiano, para expresar "las interacciones sociales que nos parezcan más relevantes para el análisis de aquellos elementos que definen nuestro objeto" (Murillo y Mena, 2006, p. 39). La representatividad se procesa así, por saturación; "de un habitus con respecto al objeto investigado" (Callejo, 2001, p. 113). El sujeto representa su condición social mediante los criterios de homologación -edad, género, estrato social, proveniencia geográfica-, que también son criterios de representatividad.

Podemos afirmar que los "fenómenos ideológicos y culturales colectivos -valores, creencias, representaciones, proyectos, es decir, la semántica colectiva de la vida socialtambién forman parte de la realidad objetiva" (Bertaux, 2005, p. 11). Este hecho nos permite formular "sujetos tipos", aquellos que producen, desde una determinada posición social, un discurso reconocible sobre el tema a analizar (Weber, 1993). Esto se debe a que "la intuición comprehensiva consiste en tener en mente las diferentes características del concepto a tipificar -indicadores típicos y sus posibles valores- $y$, mediante un esfuerzo de análisis, síntesis e interrelación múltiple, construir un todo abstracto, pero coherente" (Sánchez de Puerta, 2006, p. 22). En definitiva, este es el desafío que nos proponen los 50 grupos de discusión que formulamos porque "una de las materias primas fundamentales de la perspectiva cualitativa es el discurso: sólo a través de dispositivos conversacionales abiertos se pueden alcanzar las vivencias de los sujetos y, a partir de estas, sus estrategias" (Callejo, 1995, p. 9).

Para reconstruir un discurso social debemos centrarnos en la autoexégesis a la que se somete el sujeto durante la acción dialógica. "Tanto el sentido común como la experiencia nos llevan a rechazar simultáneamente dos posiciones demasiado categóricas: la que dice que todo lo que el sujeto cuenta en su relato es objetivamente exacto; y la que proclama, a la inversa, que nada de lo que dice el sujeto se pude dar por cierto" (Bertaux, 2005, p. 77). El análisis weberiano apela a la imaginación sociológica haciendo una "relación de hechos conocidos con puntos de vista también conocidos" (Weber, 2001, p.101).

La materialización de un número tan significativo de grupos de discusión (50 en total) acerca de la misma temática, memorias colectivas de las dictaduras y transiciones ibéricas, tenía como principal propósito captar las polifonías del recuerdo, capaces de darnos elementos significativos para que se pudieran extraer tipologías de la memoria como explicaremos en seguida. Estudiar conjuntamente las memorias colectivas de Portugal y España, países con características sociopolíticas-mediáticas similares en la lógica enunciada por Hallin y Mancini (2004), nos permite percibir qué variables pueden establecer denominadores comunes del recuerdo y a la vez plasmar especificidades reveladoras de dimensiones contextuales. Después de realizados los grupos de discusión, cuyas estrategias metodológicas detallamos en las ocho primeras notas de pie de página de este texto, pasamos a las respectivas transcripciones y análisis de los datos.

La lectura de los grupos de discusión no obedeció a una interpretación casuística de lo que se expresó en cada grupo en concreto. La decodificación que establecemos asumió los discursos como portadores de una manifestación social previa, "para comprender (y por tanto, para controlar) los significados y las funciones de los "textos" escritos o hablados, necesitamos comprender (controlar) sus contextos" (Van Dijk, 2004: 14). Demos sentido al actor como sujeto social que se construye fuera de su propio grupo de discusión. Todo ello, bajo el añadido del planteamiento hermenéutico que "ha defendido siempre la seguridad del comprehender frente al escepticismo histórico y la arbitrariedad del subjetivismo" (Dilthey, 2000, p. 199). Se trata de una traducción de los significados que construyen ese mismo discurso (Gadamer, 1989). Es decir, la traducción de la experiencia de aquellos que la vivieron, directa o indirectamente, con la responsabilidad de que toda traducción contiene problemas de interpretación. Para minimizar estos efectos debemos captar el sentido que los actores dan a sus propias acciones desde una lógica comprensiva, pues las palabras indican un sentido propio para sus acciones. 
Los discursos nos permitieron extraer etiquetas interpretativas, donde percibimos variables explicativas que determinaban lecturas del pasado, son marcos sociales de la memoria en la definición de Maurice Halbwachs (2004). De forma breve, dadas las restricciones de extensión del texto, resumimos en la Tabla 1 las principales evidencias extraídas de los grupos de discusión hechos en Portugal y España que configuran una posibilidad de tipología del recuerdo.

Tabla 1. Etiquetas resultantes del análisis de los grupos de discusión

\begin{tabular}{|c|c|c|}
\hline \multicolumn{2}{|c|}{ Marcos sociales de la memoria } & $\begin{array}{c}\text { Propuestas de lecturas de los } \\
\text { discursos }\end{array}$ \\
\hline $\left.\begin{array}{l}\text { Familia } \\
\text { Religión } \\
\text { Clase social } \\
\text { Generacionales }\end{array}\right\}$ & $\begin{array}{l}\text { Halbwachs } \\
\text { (2004) }\end{array}$ & $\begin{array}{l}\text { Procesos de socialización; } \\
\text { latentes/manifiestos }\end{array}$ \\
\hline Geográficos/Co & xtuales & Oficiales/Opositoras \\
\hline Grado de Politiz & & Politizadas/No politizadas \\
\hline
\end{tabular}

Verificamos que las premisas invocadas por el teórico francés acerca del proceso de formación de la memoria colectiva pertenecían a una aproximación macroteórica donde difícilmente conseguimos extraer evidencias sustantivas La lectura de los procesos de socialización del recuerdo no fue fácil de desvelar, pero nos dieron pistas importantísimas para que percibiéramos que la posición social, los valores sociales vigentes y la forma como son compartidas las experiencias del pasado relatado (inclusivamente las ausencias de significado de los hechos), resultaron como muy determinantes para la configuración de las percepciones de los acontecimientos. Deducimos lecturas que nos permitieron operacionalizar tipologías manejables del punto de vista empírico.

Eso nos permitió constatar lo que ya nos parecía previsible desde las lecturas de los procesos de socialización de la memoria, que el sentido del recuerdo es dependiente de la relación, directa o indirecta, con el pasado autoritario. Los que vivieron los acontecimientos de las dictaduras de forma directa construyen su percepción del pasado como expresión de una memoria vivida que gana significado en el presente. Distinta de la reconstrucción del recuerdo de las generaciones que nacieron posteriormente a los acontecimientos históricos y que incorporan el sentido del pasado "mediante las personas que participaron en los acontecimientos" (Margalit, 2002, p.44), fabricando una memoria mediada de los hechos. Las memorias vividas y mediadas son a la vez atravesadas por las memorias mediatizadas, fruto de los discursos informativos, ficcionales e históricos que circulan en el espacio público.

Fue interesante percibir que la interpretación de los relatos mediatizados estaba decididamente marcada por los procesos de socialización de la memoria, dependiendo si las lecturas del pasado estaban marcadas por posiciones más legitimadoras o críticas con las dictaduras ibéricas. Discurriendo a partir de esta evidencia percibimos que las cuestiones contextuales de donde se producen la memoria, lugares que se posicionaron de forma más beligerante o colaborativa con los valores autoritarios, eran determinantes para condicionar las lecturas, configurando memorias opositoras u oficiales.

De igual forma que observamos que la interpretación del pasado era dependiente del grado de politización de los sujetos. El tipoideal politizado (vinculado a partidos, asociaciones, colectivos sociales) construye un discurso más crítico con las formulaciones sobre los acontecimientos históricos, produciendo lecturas menos simplificadas de los acontecimientos y actores involucrados. 
De igual forma notamos que las memorias colectivas politizadas se nutrían de las experiencias y conocimiento personales/próximos y alimentaban un mayor debate social acerca del pasado (conversaciones en el seno de la familia, amigos y debate público) por contraste con los grupos despolitizados ${ }^{12}$.

Los marcos sociales de la memoria resultantes de los grupos de discusión hechos en Portugal y España denotan una posibilidad de tipificaciones del recuerdo de pasados dictatoriales, divergiendo en los niveles de beligerancia discursiva fruto de identidades políticas marcadas por la Guerra Civil española. Las tipologías identificadas nos parecen idóneas para que se apliquen en otros contextos marcados por pasados autoritarios, complementadas por un segundo ejercicio de matización que no llevamos a cabo en este texto por limitaciones de espacio ${ }^{13}$. Tal lectura debería contemplar el cruce de todas las dimensiones que configuran el puzzle del recuerdo, barajando tipologías compuestas que perfilan a la memoria colectiva como un entramado complejo de atributos sociales múltiples (vividas/mediadas/oficiales/opositoras/politizadas/no politizadas).

\section{Referencias}

Arias, M. (2002). Sociedad y lenguaje: el espacio de diálogo entre las técnicas de investigación social y la sociolingüística. Estudios de Sociolingüistica, 3(1), i-xviii.

Assmann, J. (1995). Collective memory and cultural identity. New German Critique, 65, 125-133.

Bauman, Z. (2002). La hermenéutica y las ciencias sociales. Buenos Aires: Nueva Visión.

Bertaux, D. (2005). Los relatos de vida. Perspectiva etnosociológica. Barcelona: Bellaterra.

Bloch, M. (2001). Introducción a la Historia. Madrid: FCE.

Bourdieu, P. (2001). ¿Qué significa hablar? Madrid: Akal.

Bourdieu, P. (2006). La distinción. Criterio y bases sociales del gusto. Madrid: Taurus.

Bourdieu, P. \& Wacquant, L. (eds.) (1992). An invitation to reflexive sociology. Chicago: CUP.

Bruner, J. (2003). Self-making narratives. In R. Fivush \& C. A. Haden (Eds.), Autobiographical memory and the construction of a narrative self: Developmental and cultural perspectives (pp. 209-225). The moderator should only interfere with more questions if the group is blocked. NJ: Lawrence Erlbaum Associates Publishers.

Callejo, J. (1995). La audiencia activa. El consumo televisivo: discursos y estrategias. Madrid: CIS.

Callejo, J. (2001). El grupo de discusión. Introducción a una práctica de investigación. Barcelona: Ariel.

Carriço Reis, B. (2009) De la dictadura a la democracia; recuerdos y olvidos de la transición política española. Medios de comunicación y reconstrucción de la(s) memoria(s) colectiva(s) en España. Tesis de doctorado no publicada, Universidad Rey Juan Carlos de Madrid (España).

Carriço Reis, B. (2015). Memórias em segunda mão; lembranças juvenis de um salazarismo ficcionado. In A. Barbalho e L. Marôpo (Orgs.), Infância, Juventude e Mídia: olhares lusobrasileiros (pp. 95-111). Fortaleza: Publicações da Universidade Federal do Ceará.

Crotty, M. (1998). The foundations of social research. London: Sage

Dilthey, W. (2000). Dos escritos sobre hermenéutica: El surgimiento de la hermenéutica y los Esbozos para una crítica de la razón histórica. Madrid: ISTMO.

Duero, D. (2006). Relato autobiográfico e interpretación: una concepción narrativa de la identidad personal. Atenea Digital: Revista de pensamiento e investigación social, 9, 131- 151.

Gadamer, H-G. (1989). Truth and Method. New York: Crossroad.

\footnotetext{
12 Una cuestión complementaria que nos parece interesante señalar tiene que ver con los discursos de género. Las mujeres, de cualquier edad y condición, destacan el cambio de status y roles femeninos como un profundo cambio de mentalidades, no como el resultado de un claro cambio de régimen.

${ }^{13}$ Para el efecto véase Carriço Reis (2009).
} 
Garfinkel, H. (2006). Estudios em etnometodología. Barcelona: Anthropos

Grondin, J. (2008). ¿Qué es la hermenéutica? Barcelona: Herder.

Gutiérrez, J. (2008). Dinámica del grupo de discusión. Madrid: CIS.

Gutiérrez de Álamo, F. (2009). Análisis sociológico del sistema de discursos. Madrid: CIS.

Halbwachs, M. (2004). La memoria colectiva. Zaragoza: Prensa Universitarias de Zaragoza.

Hallin, D. \& Mancini, P. (2012). Comparing Media Systems. Cambridge: Cambridge University Press.

Heller, A. (2003). Memoria cultural, identidad y sociedad civil. Indaga - Revista Internacional de Ciencias Sociales y Humanas, 1, 5-17.

Hennink, M. (2014). Focus Group Discussions. Oxford: Oxford University Press.

Hoskins, A. (Ed.) (2018). Digital memory studies. Media pasts in transition. New York: Routledge.

Ibáñez, J. (1985). Análisis sociológicos de textos o discursos. Revista Internacional de Sociología, 3, 119-160.

Laughey, D. (2006). Music and Youth Culture. Edinburgh: Edinburgh University Press.

Levy, D. \& Sznaider, N. (2002). Memory Unbound: The Holocaust and the Formation of Cosmopolitan Memory. European Journal of Social Theory, 5 (1), 87-106.

Margalit, A. (2002). Ética del recuerdo. Barcelona: Herder.

Murillo, S. \& Mena, L. (2006). Detectives y camaleones: el grupo de discusión. Una propuesta para la investigación cualitativa. Madrid: Talasa.

Ormeño, J. (2004). Algunas cuestiones teóricas relativas a la "memoria" como práctica social. Athenea Digital, 6, 31-46.

Pérez-Agote, J. (2008). Memoria colectiva y socialización: Halbwachs y los durkheimianos desde la crisis educativa de la modernidad. Revista Anthropos, 218, 85-95.

Pearson, D. \& Vossler, A. (2016). Methodological issues in focus group research: The example of investigating counsellors' experiences of working with same-sex couples. Counselling Psychology Review, 31(1).

Poggi, G. (2005). Encuentro con Max Weber. Buenos Aires: Nueva Visión.

Ricoeur, P. (1999). Historia y narratividad. Barcelona: Paidós.

Ricoeur, P. (2003). La Memoria, La Historia, El olvido. Madrid: Trotta.

Ritzer, G. (ed.) (1992). Metatheorizing. Newbury Park: Sage.

Sánchez de Puerta, F. (2006). Los tipos ideales en la práctica: significados, construcciones, aplicaciones. EMPIRIA. Revista de Metodología de las Ciencias Sociales, 11.

Schleiermacher, F. (1990). Sobre la religión: discursos a sus menospreciadores cultivados. Madrid: Tecnos.

Steiner, G. (1997). Pasión intacta. Madrid: Siruela.

Steiner, G. (2008). Os livros que não escrevi. Lisboa: Gradiva.

Vala, J. (2000). Representações sociais e psicología social do conhecimento quotidiano. In J. Vala \& M. Monteiro. (Coords.) Psicologia Social (pp. 457-496). Lisboa: Gulbenkian.

Van Dijk, T. (2004). Discurso y Dominación. Grandes Conferencias en la facultad de Ciencias Humanas de la Universidad Nacional de Colombia, No 4.

Weber, M. (1993). Economía y Sociedad. Madrid: FCE.

Weber, M. (2001). Ensayos sobre metodología sociológica. Buenos Aires: Amorrortu.

Wilkinson, S. (2004). Focus group research. In D. Silverman (ed.), Qualitative research: Theory, method, and practice (pp. 177-199). Thousand Oaks, CA: Sage.

Wittgenstein, L. (2009). Philosophical Investigation. NJ: John Wiley and Sons.

Wolf, M. (2000). Sociologías de la Vida Cotidiana. Madrid: Catedra. 